\title{
Reações adversas a medicamentos entre idosos institucionalizados: prevalência e fatores associados
}

\author{
Adverse drug reactions among institutionalized elderly: \\ prevalence and associated factors \\ Reacciones adversas a los medicamentos entre los ancianos: \\ prevalencia y factores asociados \\ Thaís Jaqueline Vieira de LIMA $^{1}$ \\ Cléa Adas Saliba GARBIN ${ }^{1}$ \\ Paula Caetano ARAÚJO ${ }^{2}$ \\ Artênio José Ísper GARBIN ${ }^{1}$ \\ Tânia Adas SALIBA ${ }^{1}$ \\ Orlando SALIBA \\ 'Departamento de Odontologia Infantil e Social, Faculdade de Odontologia de Araçatuba, \\ Universidade Estadual Paulista (Unesp), 16015-050, Araçatuba-SP, Brasil \\ ${ }^{2}$ Área de Odontologia Preventiva e Social, Faculdade de Odontologia da Universidade Federal de Uberlândia (FOUFU), \\ 38405320, Uberlândia-MG, Brasil
}

\section{Resumo}

Introdução: As reações adversas a medicamentos estão relacionadas a desfechos negativos da terapia, aumentando o risco de morbidade e mortalidade. Objetivos: Sendo os idosos pacientes altamente suscetíveis a essas reações devido a vários fatores, como as alterações fisiológicas que alteram a farmacocinética e farmacodinâmica, além do alto consumo de medicamentos devido à multiplicidade e cronicidade de doenças que apresentam, é de fundamental importância estudar a ocorrência das reações adversas a medicamentos nesta população. Material e Método: Para verificar a prevalência dessas reações entre idosos institucionalizados e analisar os fatores associados a sua ocorrência, foram analisados os prontuários médicos de 261 residentes em Instituições de Longa Permanência para Idosos e realizadas entrevistas às enfermeiras responsáveis. Resultados: Dos 253 idosos que utilizavam medicamentos diariamente, $17,4 \%$ apresentaram reações adversas, totalizando 68 ocorrências. Entre os participantes do estudo que utilizavam um medicamento potencialmente inapropriado para idoso, 95,4\% apresentaram uma reação adversa, sendo o ácido acetilsalicílico e a risperidona os medicamentos asssociados à maior prevalência de reações $(31,2 \%)$. Os fatores que apresentaram associação estatística significante com a ocorrência de reações adversas foram o sedentarismo $(\mathrm{p}=0,04)$ e uso de medicamentos potencialmente inapropriados para idosos $(\mathrm{p}=0,02)$. Conclusão: A prevalência de reações adversas entre idosos institucionalizados apontou um risco mais elevado para idosos que não praticam atividades físicas e que utilizam medicamentos potencialmente inapropriados para idosos. Hábitos nocivos como o tabagismo, apesar de constituir fator de risco para uma série de doenças crônicas, não mostrou associação estatisticamente significante com a ocorrência de reações adversas a medicamentos.

Descritores: Farmacoepidemiologia; Idoso; Uso de Medicamentos; Efeitos Colaterais e Reações Adversas Relacionados a Medicamentos.

\begin{abstract}
Introduction: The adverse drug reactions are related to negative outcomes of therapy, increasing the risk of morbidity and mortality. Objective: Being seniors patients highly susceptible to these reactions due to various factors such as the physiological changes that alter the pharmacokinetics and pharmacodynamics, and the high consumption of drugs due to multiplicity and chronicity of disorders that present, it is of fundamental importance to study the occurrence of adverse drug reactions this population. Material and Methods: To determine the prevalence this reactions among institutionalized elderly and analyze the factors associated with their occurrence, we analyzed the medical records of 261 elderly residents in long-stay institutions for the elderly and interviewed nurses responsible for them. Results: Of the 253 seniors who used drugs daily, 17,4\% showed adverse drug reactions, totaling 68 occurrences. Among of seniors who used a potentially inappropriate medication for elderly, 95,4\% had a adverse reactions, and the acetylsalicylic acid and risperidone the drugs linked with the highest prevalence of reactions $(31,2 \%)$. The factors that showed a statistically significant association with the occurrence of adverse reactions were sedentary lifestyle $(p=0,04)$ and the use of potentially inappropriate medications for elderly $(\mathrm{p}=0,02)$. Conclusion: The prevalence of adverse reactions among institutionalized elderly indicates higher risk for seniors who do not practice physical activities and who uses potentially inappropriate medications for the elderly. Harmful habits as smoking, although constitute risk factors for a number of chronic diseases, showed no statistically significant association with the occurrence of adverse drug reactions.

Descriptors: Pharmacoepidemiology; Aged; Drug Utilization; Drug-Related Side Effects and Adverse Reactions.
\end{abstract}

\section{Resumen}

Introducción: Las reacciones adversas a fármacos se relacionan con los resultados negativos de la terapia, lo que aumenta el riesgo de morbilidad y mortalidad. Objetivos: Como los ancianos son pacientes altamente susceptibles a estas reacciones debido a diversos factores, tales como los cambios fisiológicos que alteran la farmacocinética y la farmacodinámica, además del alto consumo de drogas debido a la multiplicidad y la cronicidad de las enfermedades que se presentan, es de importancia fundamental estudiar la ocurrencia de reacciones adversas a los medicamentos en esta población. Materiales y métodos: Para determinar la prevalencia de estas reacciones entre los ancianos y analizar los factores asociados a su aparición, fueron analizados los registros médicos de 261 residentes en instituciones de larga estadía para los ancianos y realizadas entrevistas con las enfermeras responsables. Resultados: De los 253 ancianos que utilizan medicamentos todos los días, 17,4\% experimentaron reacciones adversas, por un total de 68 apariciones. Entre los participantes en el estudio que usaron un medicamento potencialmente inapropiado para ancianos, 95,4\% experimentaron una reacción adversa, siendo el ácido acetilsalicílico y risperidona medicamentos asssociados la mayor prevalencia de reacciones $(31,2 \%)$. Los factores que mostraron una asociación estadísticamente significativa con la aparición de reacciones adversas fueron el sedentarismo $(\mathrm{p}=0,04)$ y el uso de medicamentos potencialmente inapropiados para los ancianos $(\mathrm{p}=0,02)$. Conclusión: La prevalencia de reacciones adversas entre los ancianos institucionalizados mostró un mayor riesgo para los ancianos que no realizan actividades físicas y que utilizan medicamentos potencialmente inapropiados para ancianos. Hábitos nocivos como el tabaquismo, aunque es un factor de riesgo para una serie de enfermedades crónicas, no mostraron asociación estadísticamente significativa con la aparición de reacciones adversas a los medicamentos.

Descriptores: Farmacoepidemiología; Anciano; Utilización de Medicamentos; Efectos colaterales y reacciones adversas relacionados con medicamentos

\section{INTRODUÇÃO}

A terapêutica medicamentosa constitui-se uma prática essencial para o tratamento e controle de inúmeras enfermidades. Entretanto, nenhum medicamento é completamente seguro. Todos, em menor ou maior grau, podem desencadear uma reação adversa a medicamentos (RAM) ${ }^{1}$. 
A Organização Mundial da Saúde (OMS) define como reação adversa a medicamento "qualquer efeito prejudicial ou indesejável, não intencional, que aparece após a administração de um medicamento em doses normalmente utilizadas no homem para a profilaxia, o diagnóstico e o tratamento de uma enfermidade" ${ }^{2}$.

De modo geral, as RAM são associadas a desfechos negativos da terapia, visto que podem aumentar de modo significativo o tempo de permanência hospitalar, ou até mesmo conduzir ao óbito. Em muitos casos, o tratamento da RAM inclui a inserção de novos medicamentos à terapêutica, elevando o risco da cascata iatrogênica ${ }^{3}$. Todos esses fatores acarretam um aumento considerável dos gastos públicos em saúde, o que ressalta sua importância no âmbito da saúde pública.

$\mathrm{O}$ idoso é especialmente vulnerável às RAM em virtude de fatores que o caracterizam: particularidades farmacocinéticas e farmacodinâmicas, presença de múltiplas doenças, uso de número elevado de medicamentos e o tipo de medicamentos prescritos ${ }^{4}$. Enfim, o envelhecimento, por si só, é um fator de risco para muitas doenças, criando a demanda por mais cuidados, o que implica o uso de grande número de medicamentos, e estes, por sua vez, aumentam o risco da ocorrência de $\mathrm{RAM}^{5}$.

Após analisar 9.793 internações hospitalares de idosos, registradas pelo Sistema de Internações Hospitalares do SUS, Paula et al. ${ }^{6}$ concluíram que há uma tendência crescente dos problemas associados ao uso de medicamentos por idosos.

Nessa problemática, Wang et al. $^{7}$ sugerem que aumentar os níveis de atividade física pode não apenas adiar o desenvolvimento de doenças crônicas, mas também gerar grande economia para os sistemas de saúde, pois protelando-se a evolução das doenças, tende-se a reduzir o número de medicamentos utilizados, minimizando a possibilidade da ocorrência de RAM. Com isso, rompe-se o ciclo que leva ao desencadeamento da RAM e suas consequências. Além disso, alguns estudos mostram que indivíduos sedentários podem ser mais propensos a consumir mais medicamentos ou gastar mais em saúde, em comparação com indivíduos que praticam atividade física ${ }^{8}$.

Visto que a maioria dos estudos sobre RAM são realizados entre pacientes hospitalizados, ressalta-se a importância deste estudo, realizado em Instituições de Longa Permanência para Idosos (ILPI), onde residem grupos especiais de idosos, cujas particularidades os diferem dos longevos que vivem na comunidade. Isso porque a institucionalização é uma situação estressante que pode desencadear, entre outros problemas, a depressão, pois leva o idoso a passar por transformações de todos os tipos. É um isolamento social que traz como consequência a perda de identidade, de liberdade, de autoestima, o que justifica a alta prevalência de transtornos psicológicos nessas instituições ${ }^{9}$.

Portanto, o idoso institucionalizado constitui, em geral, um grupo cuja morbidade apresenta-se superior à de idosos da comunidade, aumentando, desta forma, a predisponibilidade da ocorrência de efeitos/reações adversas, devido à maior necessidade de ingestão de medicamentos ${ }^{10}$.

Assim, é objetivo deste trabalho verificar a prevalência de RAM entre idosos institucionalizados de quatro municípios do estado de São Paulo e os fatores associados à sua ocorrência, a fim de evitá-la ou permitir a identificação e o tratamento precoce.

\section{MATERIAL E MÉTODO}

Trata-se de um estudo transversal, cuja população de estudo foi constituída por idosos residentes em ILPI, com idade igual ou superior a 60 anos. O período da coleta dos dados foi compreendido entre os meses de dezembro de 2011 a maio de 2012. Foram selecionados por conveniência quatro municípios do estado de São Paulo, Brasil, sendo três municípios de grande porte (Araçatuba, Botucatu e Bauru) e um de médio porte (Penápolis).

Participaram do estudo todos os idosos que residiam nas instituições pertencentes a esses municípios, totalizando seis ILPI estudadas, com exceção dos que não haviam completado 60 anos durante o período de coleta de dados e dos que não residiam nas instituições em período integral.

Os dados foram obtidos por meio da análise documental dos prontuários médicos dos idosos residentes, complementados com entrevistas dirigidas às enfermeiras responsáveis de cada instituição, realizadas por um único entrevistador.

Para direcionar a entrevista, utilizou-se um roteiro contendo perguntas abertas e fechadas, previamente testado por meio de um estudo-piloto realizado com um grupo de enfermeiras e auxiliares de uma ILPI do município de Araçatuba para analisar a aceitação da pesquisa e observar se existia dificuldade de compreensão de alguma questão. $\mathrm{Na}$ sequência foram realizadas as devidas correções e adaptações.

Foram registrados os seguintes dados: características socioeconômicas e questões relacionadas à saúde, como internações hospitalares ocorridas durante o ano, doenças, sintomas ou síndromes presentes, bem como o uso diário de medicamentos prescritos (nome e indicação do medicamento e sintomas após tomar a medicação).

As informações relacionadas aos sintomas foram obtidas por meio da questão aberta: "O paciente $\mathrm{X}$ sente alguma coisa após a tomada de determinado medicamento? Se sim, qual sintoma observado?" dirigida à enfermeira. Os prontuários médicos foram consultados para complementação das informações obtidas por meio da entrevista.

Klepper e Edwards ${ }^{11}$ apontam para consideráveis variações individuais nas respostas de entrevistados em se tratando de uma suspeita de reação adversa. Para controle dos possíveis vieses na avaliação das RAM por parte das enfermeiras entrevistadas, foram avaliadas quanto à causalidade apenas as suspeitas que foram confirmadas na análise dos prontuários. As reações suspeitas pelas enfermeiras que não tiveram seu histórico inserido no prontuário (informações quanto à data de início dos sintomas e diagnóstico confirmado pelo médico da instituição) foram desconsideradas.

Foram catalogados todos os produtos farmacêuticos prescritos ao longo do mês anterior à coleta dos dados, em que se registraram todas as informações que constavam no prontuário. Foram consideradas apenas as reações que apareceram em decorrência do uso de medicamentos prescritos durante este mesmo período.

Todos os produtos farmacêuticos industrializados e fórmulas magistrais foram incluídos no banco de dados e analisados, sendo classificados em categorias terapêuticas, de acordo com seu princípio ativo, tendo como referência o Anatomical-Therapeutical-Chemical Classification System $^{12}$. A variável dependente foi definida como a presença de pelo menos uma RAM, detectada por meio da análise dos prontuários e de entrevista à enfermeira responsável. 
Teoricamente, as RAMS são classificadas em duas grandes categorias: reações Tipo A e reações Tipo B. As reações tipo A envolvem respostas normais e exageradas, mas indesejáveis, aos fármacos em questão ${ }^{13}$. Incluem-se na categoria de RAM do tipo B as reações de intolerância ao fármaco, hipersensibilidade ou idiossincráticas ${ }^{14}$. Embora raras, estas RAM podem causar sérias morbidades e, em alguns casos, o óbito.

Cada suspeita de RAM foi avaliada segundo o Algoritmo de Naranjo ${ }^{15}$ também denominado Escala de Probabilidade de RAM, cuja aplicação resulta em uma classificação de decisão (Causalidade), que é a análise da probabilidade de um determinado fármaco ser a causa de um efeito adverso observado, ou seja, o relacionamento entre um determinado tratamento com um fármaco específico e o aparecimento de RAM $^{16}$.

Foi utilizada essa escala por ser um método simples, validado e com alta taxa de confiabilidade e reprodutibilidade. No entanto, nenhum algoritmo de causalidade desenvolvido é capaz de determinar, com certeza, que determinado evento é uma RAM ${ }^{17}$. A vantagem em utilizá-los está na padronização dos métodos em analisar os efeitos adversos, pois são instrumentos estruturados especificamente para identificação de RAM, devendo, teoricamente, fazer uma decisão mais objetiva da causalidade $^{18}$.

A análise pelo algoritmo de Naranjo classifica as RAM de acordo com as seguintes categorias: definidas (910 pontos), prováveis (5-8 pontos), possíveis (1-4 ponto) ou duvidosas (escore 0). Neste estudo, foram consideradas somente as reações definidas e prováveis. Em seguida, as RAM foram classificadas segundo a WHO Adverse Reaction Termonology- WHO-ART) ${ }^{19}$.

As variáveis independentes acerca dos comportamentos relacionados à saúde foram: a) prática de atividades físicas - definida como a prática de exercícios no mínimo uma vez por semana ${ }^{20}$; b) histórico de etilismo - foi considerado como histórico positivo para etilismo o idoso que consumia qualquer quantidade de bebida alcoólica ao ser admitido na instituição; e c) tabagismo - definiu-se como fumante aquele indivíduo que fumava diariamente, não importando a quantidade ${ }^{21}$.

As variáveis independentes sociodemográficas incluíram gênero e idade. A idade foi categorizada em $\geq 75$ e $<75$ e o gênero em "feminino" e "masculino".

Também foram incluídas as seguintes variáveis independentes: a) internações hospitalares - considerando se o indivíduo foi internado durante $\mathrm{o}$ ano anterior à pesquisa; b) dependência, cujo grau foi medido através da escala de $\mathrm{Katz}^{22}$, sendo categorizada em: "sim" (considerando os idosos classificados como dependentes ou parcialmente dependentes) e "não" (idosos classificados como independentes pela escala); c) polifarmácia determinada mediante a contagem dos medicamentos usados concomitantemente, utilizando o ponto de corte de cinco ou mais medicamentos ${ }^{23}$; d) polimorbidade - paciente acometido por quatro ou mais doenças ${ }^{23}$ e e) uso de medicamento potencialmente inapropriado (MPI) para idosos cuja identificação foi realizada por meio dos critérios de Beers atualizados ${ }^{24}$.

Inicialmente, foram realizadas análises bivariadas para avaliação isolada do efeito de cada variável. P-valores foram gerados utilizando o teste de associação Quiquadrado e exato de Fischer. Em seguida, foi aplicada a técnica de regressão logística múltipla para conhecer os fatores associados à ocorrência de RAM e eliminar possíveis fatores de confundimento. Para constituir o modelo de regressão foram selecionadas as variáveis que apresentaram valor de $p \leq 20 \%$ nas análises univariadas, que foram: tabagismo, prática de atividade física e utilização de MPI.

A medida de associação estimada foi a razão de chances ou odds ratio (OR) e o nível de significância adotado foi de $5 \%$. Todas as análises foram conduzidas utilizando o software estatístico BioEstat, versão 5.3 ${ }^{25}$.

O projeto foi aprovado pelo Comitê de Ética em Pesquisa em Seres Humanos da UNESP- Universidade Estadual Paulista - Faculdade de Odontologia de Araçatuba, com o parecer $n^{\circ}$ 01988/2011. Além disso, obteve-se a autorização dos diretores de cada instituição e o termo de consentimento informado de todos os sujeitos envolvidos.

\section{RESULTADOS}

De um total de 268 idosos, 3 não residiam na instituição, visto que ficavam apenas durante o dia com os outros internos e 4 não tinham completado até o período do estudo a idade de 60 anos, resultando em 261 participantes.

Da seleção final $(n=261) 42,5 \%$ incluíam idosos do gênero masculino e $57,5 \%$ do gênero feminino, com uma média de 77 anos de idade.

A Tabela 1 mostra que $24,1 \%$ e $23,4 \%$ eram idosos com grau parcial e total de dependência, respectivamente. A maioria $(74,7 \%)$ não praticava nenhum tipo de atividade física, e eram considerados, portanto, sedentários. Quanto aos hábitos nocivos de saúde, 39,5\% eram fumantes e $31,4 \%$ tinham histórico de etilismo antes de ser admitido na instituição.

Faziam uso contínuo de medicamentos $96,9 \%$ dos idosos participantes do estudo, sendo uma média de 5,7 medicamentos utilizados diariamente.

Entre os idosos que utilizavam medicamentos diariamente $(\mathrm{n}=253)$, houve 68 ocorrências de RAM. A média por paciente, considerando apenas os 44 idosos $(17,4 \%)$ que apresentaram RAM, foi igual a 1,5 (mínimo 1, máximo 3).

$\mathrm{O}$ número de RAMs classificadas como definidas pela Escala de probabilidade de RAM $^{16}$ foram $4[5,9 \%$ (4/68)] e 64 [94,1\% (64/68) prováveis, sendo a maioria das RAMs classificadas como desordens do sistema gastrointestinal (50\%), como pode ser visto na Tabela 2.

$\mathrm{O}$ consumo de medicamentos inapropriados para uso em idosos foi alto - mais de $80 \%$ dos idosos que utilizavam medicamentos diariamente, recebiam no mínimo um medicamento potencialmente impróprio (MPI) para idoso, sendo os antipsicóticos $(26,5 \%)$ e os analgésicos $(15,1 \%)$ os mais frequentemente prescritos e utilizados entre os medicamentos que devem ser evitados em idosos. Ansiolíticos (12,5\%), antiarrítmicos (7,2\%) e antidepressivos $(6,8 \%)$ foram encontrados em mais de 30 prescrições.

A maioria dos idosos que fazia uso de MPI, apresentou uma RAM (95,4\%). Apenas 2 idosos (4,5\%) que apresentaram RAM não utilizava MPI. Entre os medicamentos que provocaram RAM, o ácido acetilsalicílico e a risperidona foram os medicamentos asssociados à maior prevalência de RAM (31,2\%). Considerando o princípio ativo, dos 51 MPI utilizados, $54,9 \%$ estiveram associados à ocorrência de RAM.

Para análise das associações foi considerada a ocorrência de no mínimo uma RAM. Na análise bivariada foram selecionadas as variáveis que apresentaram 
significância estatística de $20 \%$, ou seja, valor de $p \leq 0,20$, que foram o tabagismo $(\mathrm{p}=0.132)$, a prática de atividade física ( $\mathrm{p}=0.013)$ e o uso de MPI $(\mathrm{p}=0.132)$. (Tabela 3$)$.

Após análise multivariada, as variáveis sedentarismo (não pratica atividade física) ( $\mathrm{p}=0,04$ - OR 0,4889- IC: 0,24;
$0,98)$ e uso de medicamentos inapropriados para idosos $(\mathrm{p}=0,02$ - OR 3,5328- IC:1,20;10,43) permaneceram fortemente associadas à ocorrência de RAM. Entretanto, a variável tabagismo perdeu significância nesta etapa da análise, conforme dados demonstrados na Tabela 4.

Tabela 1. Características dos idosos e prevalência de comportamentos relacionados à saúde

\begin{tabular}{|c|c|c|}
\hline Variável & $\mathbf{n}$ & $\%$ \\
\hline \multicolumn{3}{|l|}{ Idade(anos) } \\
\hline $60-74$ & 110 & 42,2 \\
\hline $75-84$ & 93 & 35,6 \\
\hline$=85$ & 58 & 22,2 \\
\hline \multicolumn{3}{|l|}{ Gênero } \\
\hline Masculino & 111 & 42,5 \\
\hline Feminino & 150 & 57,5 \\
\hline \multicolumn{3}{|l|}{ Grau de dependência } \\
\hline Independente & 137 & 52,5 \\
\hline $\begin{array}{l}\text { Parcialmente } \\
\text { dependente }\end{array}$ & 63 & 24,1 \\
\hline Dependente & 61 & 23,4 \\
\hline \multicolumn{3}{|l|}{ Tabagismo } \\
\hline Não fumante & 158 & 60,5 \\
\hline Fumante & 103 & 39,5 \\
\hline \multicolumn{3}{|l|}{ Histórico de etilismo } \\
\hline Sim & 82 & 31,4 \\
\hline Não & 179 & 68,6 \\
\hline \multicolumn{3}{|c|}{ Prática de atividade física } \\
\hline Sim & 66 & 25,3 \\
\hline Não & 195 & 74,7 \\
\hline \multicolumn{3}{|l|}{ Polifarmácia } \\
\hline Sim & 192 & 73,6 \\
\hline Não & 69 & 26,4 \\
\hline \multicolumn{3}{|c|}{ Internações Hospitalares } \\
\hline Sim & 34 & 13 \\
\hline Não & 227 & 87 \\
\hline \multicolumn{3}{|l|}{ Polimorbidade } \\
\hline Sim & 44 & 16,9 \\
\hline Não & 217 & 83,1 \\
\hline \multicolumn{3}{|l|}{ Uso de MPI } \\
\hline Sim & 209 & 80 \\
\hline Não & 52 & 20 \\
\hline
\end{tabular}

Tabela 2. Distribuição absoluta e percentual das reações adversas a medicamentos, classificadas segundo o WHO-ART

\begin{tabular}{cccc}
\hline Código & Doença & $\mathbf{n}$ & $\%$ \\
\hline 0600 & $\begin{array}{c}\text { Desordens do sistema gastrointestinal } \\
\text { Desordens do sistema nervoso }\end{array}$ & 34 & 50 \\
0420 & autônomo & 13 & 19,1 \\
0431 & Desordens da visão & 9 & 13,2 \\
& Desordens do sistema & 9 & 13,2 \\
0200 & musculoesquelético & 9 & 3 \\
0410 & Desordens do SNC e periférico & 2 & 1,5 \\
1100 & Desordens do sistema respiratório & 1 & 100 \\
Total & & $\mathbf{6 8}$ & \\
\hline
\end{tabular}

Tabela 3. Análise bivariada dos fatores associados à ocorrência de reações adversas a medicamentos (RAM)

\begin{tabular}{lccc}
\hline Variáveis & $\begin{array}{c}\text { Idosos sem RAM } \\
(\mathrm{n}=209)\end{array}$ & $\begin{array}{c}\text { Idosos com RAM } \\
(\mathrm{n}=44)\end{array}$ & $p=$ valor $^{\star}$ \\
\hline Tabagismo & & & \\
$\quad$ Fumante & 78 & 21 & 0,132 \\
$\quad$ Não-fumante & 131 & 23 & \\
$\begin{array}{l}\text { Prática de atividades } \\
\text { físicas }\end{array}$ & & & \\
$\quad$ Sim & 48 & 18 & 0,013 \\
Não & 161 & 26 & \\
Utilizam MPI & & & \\
$\quad$ Sim & 167 & 42 & 0,132 \\
Não & 40 & 4 & \\
\hline
\end{tabular}

* $p$ valor (teste qui-quadrado); $p=$ significância estatística na regressão logística múltipla; $\mathrm{OR}=$ odds ratio ou razão de chances; $\mathrm{IC}=$ intervalo de confiança
Tabela 4. Análise multivariada dos fatores associados à ocorrência de reações adversas a medicamentos (RAM)

\begin{tabular}{lccc}
\hline Variáveis & $\begin{array}{c}\text { Idosos sem RAM } \\
(\mathrm{n}=209)\end{array}$ & $\begin{array}{c}\text { Idosos com } \\
\text { RAM }(\mathrm{n}=44)\end{array}$ & $\boldsymbol{p}=$ valor $^{*}$ \\
\hline Tabagismo & & & \\
$\quad$ Fumante & 78 & 21 & 0,132 \\
$\quad$ Não-fumante & 131 & 23 & \\
$\begin{array}{l}\text { Prática de atividades } \\
\text { físicas }\end{array}$ & & & \\
$\quad$ Sim & 48 & 18 & 0,013 \\
$\quad$ Não & 161 & 26 & \\
Utilizam MPI & & & \\
$\quad$ Sim & 167 & 42 & 0,132 \\
$\quad$ Não & 40 & 4 & \\
& & & \\
\hline
\end{tabular}

\section{DISCUSSÃO}

Verificou-se que apenas 25,3\% dos idosos praticavam atividade física, resultado similar ao estudo de Silva et al. $2012^{26}$, realizado com idosos que viviam no Ceará e inferior ao nível de atividade física entre idosas de São Caetano do Sul, uma vez que, $84,9 \%$ das participantes eram ativas ${ }^{5}$. Essa discrepância pode ser explicada devido a presença de desigualdade regionais, pois, na região Sul, os idosos são mais ativos e possuem condição funcional superior aos idosos de outras regiões do país ${ }^{27}$.

O tabagismo foi verificado entre $39,5 \%$ dos participantes, diferente do encontrado por Silva et al..$^{5} \mathrm{em}$ 
que $85,6 \%$ das idosas eram tabagistas. Destaca-se aqui o fato de que em algumas das instituições do presente estudo, proibe-se o vício do fumo. Dessa forma, alguns idosos eram tabagistas antes de se tornarem institucionalizados e, foram obrigados abandonarem o vício após admissão na instituição. Talvez por isso a porcentagem de tabagistas entre os idosos institucionalizados foi menor quando comparada a idosos não institucionalizados.

Quanto ao etilismo, 31,4\% tinham esse hábito nocivo antes de serem admitidos na instituição. Salienta-se o fato de que o etilismo é proibido em todas as ILPI, diferente do tabagismo. Dessa forma, $100 \%$ dos idosos não consumiam bebida alcoólica, mas tinham histórico de etilismo.

Quase a totalidade dos idosos participantes do presente estudo utilizavam medicamentos de uso contínuo (96,9\%), resultados superiores aos encontrados em estudos realizados com idosos não-institucionalizados ${ }^{28}$. Não é de surpreender que fosse encontrada essa diferença, já que os idosos institucionalizados têm predisposição a um maior número de doenças. Porém, o consumo médio de medicamentos utilizados pelos idosos do presente estudo, apesar de ser considerado alto (média $=5,7 /$ dia) parece ser inferior ao de outros estudos realizados em instituições de outros países ${ }^{29}$. Verifica-se, portanto, que a polifarmácia é uma realidade que se faz presente não só em instituições brasileiras como também no exterior.

Dentre os participantes do presente estudo, 17,4\% foram acometidos por RAM, resultados semelhantes aos verificados por Rozenfeld et al. ${ }^{30}$, cuja ocorrência de RAM foi de $16 \%$ em um hospital público do Rio de Janeiro. Porém, a identificação das reações foi realizada através de critérios rastreadores, como o uso de antieméticos, interrupção abrupta de medicamentos e sedação excessiva.

Tangiisuran et al. ${ }^{31}$ estimaram em $13,2 \%$ a proporção de RAM em idosos com idade acima de 80 anos em um hospital universitário da Inglaterra, através de um estudo observacional, resultado inferior ao encontrado neste estudo.

Entretanto, Carvalho-Filho et al. ${ }^{32}$ observaram que, do total de complicações iatrogênicas em idosos internados num hospital universitário, $32 \%$ eram ocasionadas por medicamentos. Uma alta prevalência de RAM também foi encontrada por Passarelli et al. ${ }^{33}$, que estimaram em $62 \%$ a proporção de idosos internados em hospital universitário devido às RAM. Romeu et al. ${ }^{34}$ por meio de um estudo retrospectivo, que utilizou como fonte secundária, ficha de notificação de suspeita de reação adversa a medicamentos do sistema de farmacovigilância em um hospital de Fortaleza-Ceará, encontraram 204 reações adversas, resultado também superior ao encontrado no presente estudo $(\mathrm{n}=68)$.

Essa diferença pode ser explicada pelo fato de que no ambiente hospitalar as reações são mais graves e os registros mais acurados, diferente do encontrado nas ILPI, cuja investigação quanto à ocorrência de RAM foi baseada na análise do prontuário dos idosos residentes e entrevistas às enfermeiras ${ }^{10}$. Apesar do constante cuidado dessas profissionais, é possivel que ocorram subnotificações, visto que o paciente internado é sistematicamente monitorado, identificando-se um maior número possível de potenciais reações. Além disso, também houve discrepâncias quanto aos métodos de identificação das RAM, o que pode ter contribuído para a diferença entre os resultados encontrados.

A menor prevalência de RAM obtida na população estudada deve considerar também as limitações do algoritmo de Naranjo, que embora seja o mais utilizado, exige maior conhecimento farmacológico por parte do avaliador. No entanto, com a disponibilidade atual de informações na internet ou em livros de farmacologia/toxicologia, não existe grande dificuldade à aplicação deste algoritmo ${ }^{35}$.

Outra limitação deve-se à subjetividade, baseada na experiência clínica, que não pode ser contemplada nos algoritmos e que contribui enormemente para o estabelecimento da relação causal. Desta forma, deve-se considerar que sua maior utilidade reside nos estudos de avaliação da toxicidade de fármacos individuais, havendo assim limitação para avaliação das RAMs nos casos que envolvem a polifarmácia ${ }^{36}$.

Essas limitações, entretanto, não invalidam o algoritmo proposto para avaliação de RAMs e, sua aplicação dentro das situações rotineiras tem sido uma ferramenta importante para o diagnóstico e tratamento adequados das RAMs. Além disso, a atribuição de pontos a cada critério traz algumas vantagens, pois permite a comparação entre scores de dois ou mais fármacos suspeitos e a medição da reprodutibilidade. Também estabelece dados de incidência mais acurados, facilitam as atividades epidemiológicas e de monitoramento e a tomada de decisão ${ }^{37}$.

Quanto à comparação entre os gêneros masculino e feminino, apesar do fato de que mulheres idosas consomem mais medicamentos ${ }^{38}$, estando mais expostas à utilização de MPI, e consequentemente, apresentariam mais reações adversas a medicamentos, não houve diferença estatisticamente significativa entre homens e mulheres que apresentaram RAM no presente estudo.

Por outro lado, o uso de medicamentos potencialmente inapropriados (MPI) para idosos apresentou associação com a ocorrência de RAM de forma estatisticamente significativa, em concordância com os resultados de outros autores ${ }^{39}$.

Os MPIs associados à maior prevalência de RAM foram o ácido acetilsalicílico(AAS) e a risperidona, pois o AAS aumenta o risco de hemorragia e úlcera péptica em grupos de alto risco, que são aqueles com idade superior a 75 anos ou que utilizam corticosteróides, anticoagulantes ou antiplaquetários. Já a risperidona deve ser evitada pois aumenta o risco de acidente cerebrovascular e pode levar a óbito pessoas com demência ${ }^{24}$.

Outro fator ligado à ocorrência de RAM foi o sedentarismo, associação que pode ser explicada pelo fato de que pessoas sedentárias estão mais predispostas a doenças crônicas, principalmente as cardiovasculares, necessitando de um alto consumo de medicamentos, e consequentemente, aumentando os riscos da ocorrência de $\mathrm{RAM}^{40}$.

Uma detalhe importante que não pode ser descartado é o fato de que, apesar do emprego de métodos estatísticos (análise multivariada) que supostamente eliminem possíveis confundimentos, a condução da análise pode levar a essa ocorrência. Isso pode ser evidenciado pela predominância de medicamentos atuantes sobre o SNC. Dessa forma, o tipo e não o número de medicamentos poderia ser associado ao sedentarismo.

Apesar da importância do presente estudo, deve-se levar em conta a limitação do mesmo, visto que não é possível a extrapolação dos dados obtidos para toda população de idosos. Dessa forma, pode não ser representativo para idosos vivendo em instituições de outras regiões do país. Porém, apesar das limitações, este estudo 
identificou importantes ocorrências de RAM em ILPI, locais ainda pouco estudados acerca da farmacoterapia utilizada por idosos.

\section{CONCLUSÃO}

Concluiu-se que as reações adversas a medicamentos são problemas presentes entre os idosos residentes nas Instituições de Longa Permanência analisadas e que, hábitos nocivos como o tabagismo, apesar de constituir-se como fator de risco para uma série de doenças crônicas, não influenciou a ocorrência de RAM. Por outro lado, o sedentarismo constituiu-se fator fortemente significativo.

Diante da situação exposta, é imprescindível que os profissionais de saúde responsáveis pela atenção à saúde dos idosos estejam devidamente preparados para realizar uma prescrição medicamentosa adequada, considerando o estado clínico geral de cada paciente, evitando o uso de medicamentos impróprios para idosos e outros fatores que aumentam a possibilidade da ocorrência de reações adversas. Salienta-se a necessidade de estimular os profissionais para um monitoramento constante e reavaliação contínua dos resultados terapêuticos visando à correção de problemas associados ao uso de medicamentos por idosos.

\section{APOIO FINANCEIRO}

Pesquisa subvencionada pela FAPESP - Fundação de Amparo à Pesquisa do Estado de São Paulo, processo número 2011/12525-5.

\section{REFERÊNCIAS}

1. Machado-Alba JE, Londoño-Builes MJ, EcheverriCataño LF, Ochoa-Orozco SA. Adverse drug reactions in Colombian patients, 2007-2013: analysis of population databases. Biomedica. 2016;36(1):59-66.

2. Organização Mundial da Saúde. A Importância da farmacovigilância. Brasília: Organização PanAmericana da Saúde; 2005.

3. Batel Marques F, Penedones A, Mendes D, Alves C. A systematic review of observational studies evaluating costs of adverse drug reactions. Clinicoecon Outcomes Res. 2016; 24(8):413-26.

4. Teka F, Teklay G, Ayalew E, Teshome T. Potential drug-drug interactions among elderly patients admitted to medical ward of Ayder Referral Hospital, Northern Ethiopia: a cross sectional study. BMC Res Notes. 2016; 9(1):431.

5. Silva LJ, Azevedo MR, Matsudo S, Lopes GS. Association between levels of physical activity and use of medication among older women. Cad Saúde Pública. 2012; 28(3):463-71.

6. Paula TC, Bochner R, Montilla DER. Análise clínica e epidemiológica das internações hospitalares de idosos decorrentes de intoxicações. Rev Bras Epidemiol. 2012;15(4):828-44.

7. Wang W, McGreevey WP, Fu C, Zhan S, Luan R, Chen $\mathrm{W}$, et al. Type 2 diabetes mellitus in China: a preventable economic burden. Am J Manage Care. 2009; 15(9):593-601.

8. Anderson LH, Martinson BC, Crain AL, Pronk NP, Whitebird RR, O'Connor PJ, et al. Health care charges associated with physical inactivity, overweight, and obesity. Prev Chronic Dis. 2005;2(4):A09.

9. Tabue Teguo M, Simo-Tabue N, Stoykova R, Meillon $\mathrm{C}$, Cogne M, Amiéva $\mathrm{H}$, et al. Feelings of loneliness and living alone as predictors of mortality in the elderly: the PAQUID study. Psychosom Med. 2016;78(8):904-9.

10. Rozenfeld S. Agravos provocados por medicamentos em hospitais do Estado do Rio de Janeiro, Brasil. Rev Saúde Pública. 2007;41(1):108-15.

11. Klepper MJ, Edwards B. Individual case safety reports: how to determine the onset date of an adverse reaction. Drug Saf. 2011; 34(4):299-305.

12. Anatomical Therapeutic Chemical Classification. Index with defined daily doses (DDDs). 2015. Disponível em: www.whocc.no/atc_ddd_index. Acesso em: 14 set. 2016.

13. Rawlins MD, Thomas SHL. Mechanisms of adverse drug reactions. In: Davies DM, Ferner RE, De Glanville $\mathrm{H}$ (editors). Davies's textbook of adverse drug reactions. Londres: Chapman \& Hall Medical; 1991. p. 18-45.

14. Munir NG, Howard EG, Scott AW. Adverse drug reactions: a review. Drug Inform J. 1998;32(2):323-38.

15. Naranjo CA, Busto U, Sellers EM, Sandor P, Ruiz I, Roberts EA, et al. A method for estimating the probability of adverse drug reactions. Clin Pharmacol Ther. 1981;30(2):239-45.

16. World Health Organization. Uppsala monitoring centre. Programme for international drug monitoring. 2010. Disponível em: www.who-umc.org/ DynPage.aspx $? \mathrm{id}=13140 \& \mathrm{mn}=1514 \# 3$. Acesso em: 17 maio 2011.

17. Cochrane ZR, Hein D, Gregory PJ. Medication misadventures: adverse drug reactions and medication errors. In. Malone PM, Kier K, Stanovich JE, Malone MJ (editors). Drug information: a guide for pharmacists. USA: MC Graw-Hill; 2001. p. 599 - 634.

18. Doherty MJ. Algorithms for assessing the probability of an Adverse Drug Reaction. Respiratory Medicine CME. 2009;2(2):63-7..

19. World Health Organization. The WHO Adverse Reaction Terminology WHO-ART. 2015. Disponível em: www.umc-products.com/ DynPage.aspx? id=4918. Acesso em: 14 set. 2016.

20. Lima MG, Barros MBA, César CLG, Goldbaum M, Carandina L, Alves MCGP. Health-related behavior and quality of life among the elderly: a population-based study. Rev Saúde Pública. 2011;45(3):485-93.

21. Marcopito LF, Rodrigues SSF, Pacheco MA, Shirassu MM, Goldfeder AJ, Moraes MA. Prevalência de alguns fatores de risco para doenças crônicas na cidade de São Paulo. Rev Saúde Pública. 2005;39(5):738-45.

22. Katz S, Downs TD, Cash HR, Grotz RC. Progress in development of the index of ADL. Gerontologist. 1970;10(1):20-30.

23. Wawruch M, Fialova D, Zikavska M, Wsolova L, Jezova D, Kuzelova M, et al. Factors influencing the use of potentially inappropriate medication in older patients in Slovakia. J Clin Pharm Ther. 2008;33(4):381-92.

24. By the American Geriatrics Society 2015 Beers Criteria Update Expert Panel. American Geriatrics Society updated Beers Criteria for potentially inappropriate medication use in older adults. J Am Geriatr Soc. 2015;63(11):227-46.

25. Ayres M, Júnior MA, Ayres DL, Santos AAS. BioEstat: aplicações estatísticas nas áreas das ciências biomédicas. Belém: MCT/CNPq; 2007.

26. Silva GOB, Gondim APS, Monteiro MP, Frota MA, Meneses ALL. Uso de medicamentos contínuos e fatores associados em idosos de Quixadá, Ceará. Rev Bras Epidemiol. 2012; 15(2):386-95. 
27. Instituto Brasileiro de Geografia Estatística: Indicadores sociodemográficos e de saúde no Brasil. 2009. Disponível em: www.ibge.gov.br/home/ estatistica/populacao

/indic_sociosaude/2009/com_sobre.pdf. Acesso em: 13 nov. 2013.

28. Marin MJS, Cecílio LCO, Perez AEWUF, Santella F, Silva CBA, Filho JRG, et al. Caracterização do uso de medicamentos entre idosos de uma unidade do Programa Saúde da Família. Cad Saúde Pública. 2008; 24(7):1545-55.

29. Hosia-Randell HM, Muurinen SM, Pitkälä KH. Exposure to potentially inappropriate drugs and drugdrug interactions in elderly nursing home residents in Helsinki, Finland: a cross-sectional study. Drugs Aging. 2008;25(8):683-92.

30. Rozenfeld s, Chaves SMC, Reis LGC, Martins M, Travassos C, Mendes W. Efeitos adversos a medicamentos em hospital público: estudo piloto. Rev Saúde Pública. 2009;43(5):887-90.

31. Tangiisuran B, Davies JG, Wright JE, Rajkumar C. Adverse drug reactions in a population of hospitalized very elderly patients. Drugs Aging. 2012;29(8):669679.

32. Carvalho-Filho ET, Saporetti L, Souza MAR, Arantes ACLQ, Vaz MYKC, Hojaiji NHSL, et al. Iatrogenia em pacientes idosos hospitalizados. Rev Saúde Pública. 1998;32(1):36-42.

33. Passarelli MCG, Jacob-Filho W, Figueras A. Adverse drug reactions in an elderly hospitalised population. Drugs Aging. 2005;22(9):767-77.

34. Romeu GA, Távora MRF, Costa AKM, Souza MOB, Gondim ASG. Notificação de reações adversas em um hospital sentinela de Fortaleza - Ceará. R Bras Farm Hosp Serv Saúde. 2011; 2(1):5-9.

35. Cruciani JO, Troster EJ, Sousa-Marques HH. Avaliação de reação adversa a medicamentos em um caso de confusão mental. Pediatria (São Paulo) 2007; 29(3):232-6.

36. Rozenfeld S. Farmacovigilância: elementos para a discussão e perspectivas. Cad Saúde Pública. 1998;14(2):237-63.

37. Gomes MJVM, Reis AMM. Ciências farmacêuticas: uma abordagem em farmácia hospitalar. São Paulo: Atheneu; 2001.

38. Rozenfeld S. Prevalência, fatores associados e mau uso de medicamentos entre os idosos: uma revisão. Cad Saúde Pública. 2003; 19(3):717-24.

39. Varallo FR. Internações hospitalares por Reações Adversas a Medicamentos (RAM) em um hospital de ensino [dissertação] Araraquara: Faculdade de Odontologia de Araraquara da UNESP; 2010.

40. Lima TJV, Garbin CA, Garbin AJ, Sumida DH, Saliba O. Potentially inappropriate medications used by the elderly: prevalence and risk factors in Brazilian care homes. BMC Geriatr. 2013;13:52 doi: 10.1186/14712318-13-52.

\section{CONFLITO DE INTERESSES}

Os autores declaram não haver conflitos de interesse.

\section{AUTOR PARA CORRESPONDÊNCIA}

\section{Cléa Adas Saliba Garbin}

cgarbin@foa.unesp.br

Submetido em 06/01/2017 Aceito em 26/01/2017 